\title{
Advanced Abdominal Pregnancy with a Full-Term Live Fetus: Case Report
}

\author{
Mahbuba $^{1}, \mathrm{~K}_{\text {Fatema }}{ }^{2}, \mathrm{RK} \mathrm{Saha}^{3}$
}

\begin{abstract}
:
Advanced abdominal pregnancy (AAP) with a viable full-term fetus is a rarity that a few obstetricians encounter during their professional carrier. Usually it has a dramatic and catastrophic consequence both for the fetus $\&$ the mother; rather subsequent delivery of a viable full term fetus is exceptional. It is difficult to diagnose preoperatively. Though relatively rare, we received an AAP at term with live fetus in Faridpur Medical College \& hospital. Illiteracy, poverty and lack of antenatal care had resulted in her late presentation. After laparotomy the diagnosis was confirmed, a healthy male baby was delivered.
\end{abstract}

Key words: Ectopic gestation, abdominal pregnancy, advanced abdominal pregnancy, conservative management, laparotomy, placenta removal

\section{Introduction :}

Advanced abdominal pregnancy (AAP) means any extra uterine pregnancy found within the peritoneal cavity that is greater than 20 weeks gestation ${ }^{1}$. About $2 \%$ of all pregnancies are ectopic and abdominal pregnancy accounting for $1-4 \%$ of all ectopic pregnancies $^{2}$. The incidence is high in women of developing countries probably due to low socioeconomic status, high ratio of pelvic inflammatory disease or pelvic infection, history of infertility, tubal sterilization, tubal reconstruction surgery, pregnancy with intra uterine device ${ }^{3,4}$. The risk of dying from abdominal pregnancy is 7.7 times greater than from a tubal pregnancy and 90 times higher than an intrauterine pregnancy. The maternal mortality rate is estimated to be $0.5-18 \%$ and perinatal mortality rate is $40-95 \%{ }^{3}$. Morbidity is higher resulting in bleeding, anaemia, infection, preeclampsia, disseminated intravascular coagulopathy (DIC), pulmonary embolism, fistula formation $\mathrm{etc}^{5}$.

1. Dr. Mahbuba, MBBS, FCPS (Obst \& Gynae), Associate Professor $\&$ Head, Department of Obstetrics and Gynaecology, Faridpur Medical College, Faridpur.

2. Dr. Kaneez Fatema, MBBS, MS (Obst \& Gynae), Assistant Professor, Department of Obstetrics and Gynaecology, Faridpur Medical College, Faridpur.

3. Dr. Ratan Kumar Saha, MBBS, FCPS (Surgery), Assistant Professor, Department of Surgery, Faridpur Medical College, Faridpur.

\footnotetext{
Address of correspondence :

Dr. Kaneez Fatema, MBBS, MS (Obst \& Gynae), Assistant Professor, Department of Obstetrics and Gynaecology, Faridpur Medical College, Faridpur. Phone: +88-0171-167362;

E-mail: moyourakkhi2003@yahoo.com
}

An abdominal pregnancy still remains a diagnostic and therapeutic challenge for every obstetrician as it causes comparatively few symptoms. Ultrasound scan should reveal fetal body located outside the uterus as is the ectopic placenta and close approximation of fetal parts with maternal abdominal wall. Radiography should reveal overlapping of maternal spine by fetal small parts in the lateral film and maternal intestine shadows intermingling with fetal parts in the antero-posterior view $^{6}$. Now a day CT scan and magnetic resonance imaging (MRI) have been successfully used making an accurate pre-operative diagnosis ${ }^{7}$.

About $21 \%$ of babies born after an extrauterine abdominal pregnancy have birth defects, presumably due to compression of the fetus in the absence of the amniotic fluid buffer. Typical deformities include limb defects, facial and cranial asymmetry, joint abnormalities and central nervous system malformation ${ }^{8}$.

Conservative management should include inpatient care until delivery as well as serial sonography to ensure adequate fetal growth and liquor volume. Delivery is by elective laparotomy at a gestational age as close as possible to term. The management of the placenta at the time of laparotomy is of importance. It is recommended that the placenta be removed only if its entire blood supply can be ligated and that partial removal of the placenta is most hazardous and should not be undertaken'. 


\section{Case report:}

Asma, a 25 year old housewife was admitted to Faridpur Medical College Hospital from Rajbari when she had 38 weeks of pregnancy and mild lower abdominal pain. She was gravida 3rd, para 2, had one alive child of five years old and $\mathrm{H} / \mathrm{O}$ one intra uterine death both delivered vaginally. In history, it was spontaneous conception, unsure of dates having irregular antenatal checkup. At the end of first trimester she had attacks of lower abdominal pain for which she consulted a physician and an USG at 12 weeks which commented of ectopic pregnancy at right ovary with gross enlargement. Three ultra-sonogram reports done in different time revealed intrauterine pregnancy with severe oligohydramnios. As the condition of the patient was good she was treated conservatively by some general physician. At 30 weeks, she had another USG

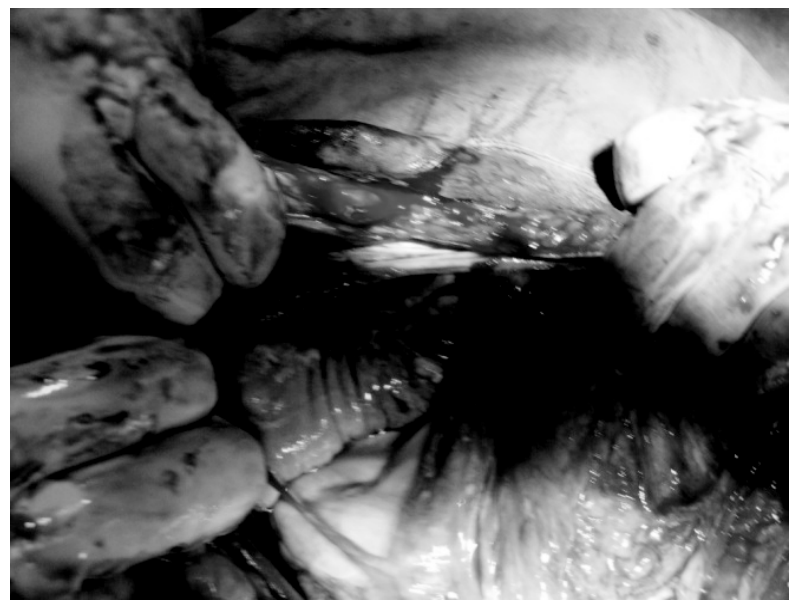

Figure -1: Baby delivered by laparotomy

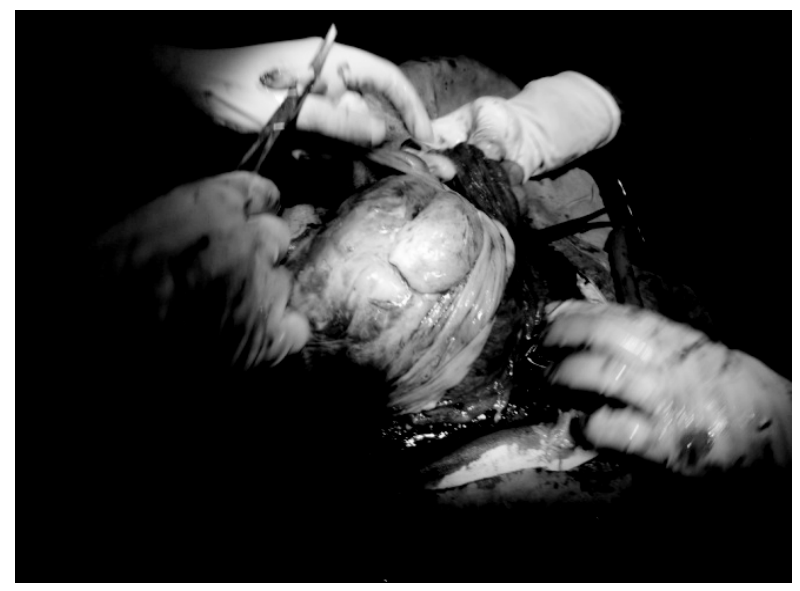

Figure -3: Placenta was attached to the omentum and gut done which commented to be an extra uterine pregnancy but the patient did not consult with an obstetrician but she had persistent mild abdominal discomfort. Finally at 38 weeks an USG revealed 34 weeks live extra uterine pregnancy with IUGR. On admission her general health was average, mildly anaemic, and normotensive. Her abdomen was slightly tender; foetal parts were superficially palpable, foetal movements were clearly seen and foetal heart sound was audible and the foetus was presented by breech. A firm mass was palpable besides the foetus. On per vaginal examination cervix was tubular and closed.

On the next day, 3 units of blood were arranged prior to operation and laparotomy was done under general anesthesia. The abdomen was opened by lower right paramedian incision carefully to avoid incision in to placentae. The membranes were not identified and there was no haemoperitoneum.

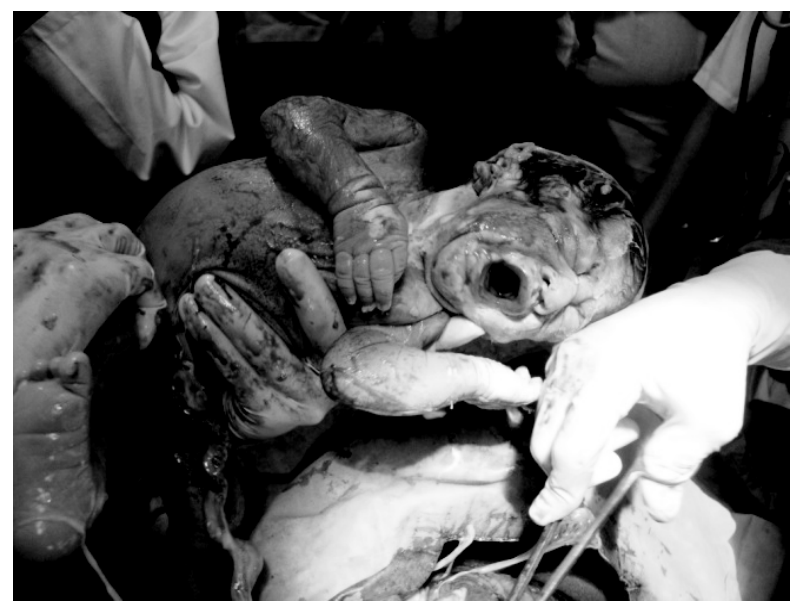

Figure - 2: Baby crying just after delivery

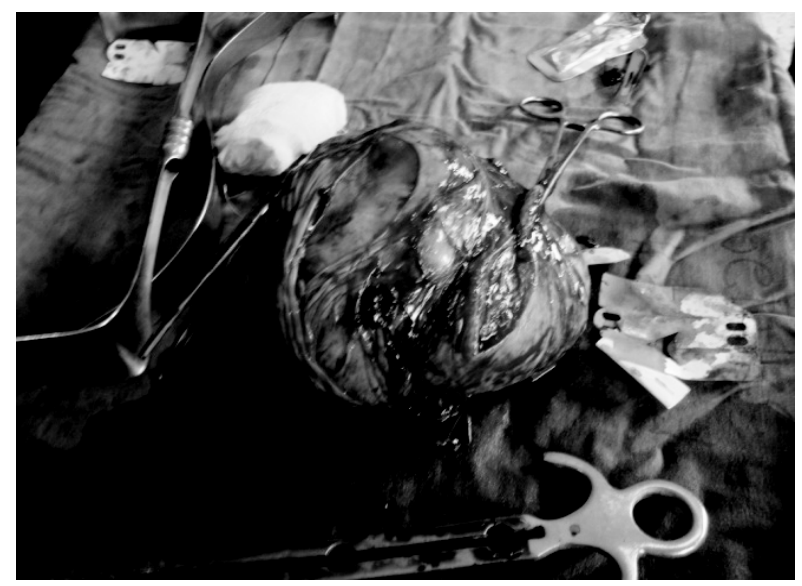

Figure -4: Placenta was removed totally 
A full-term alive male baby was found in the abdominal cavity covered by omentum and delivered with $2.5 \mathrm{~kg}$ birth weight, Apgar score 8 at one minute and 10 at 5 minute with the exception of reverse talepes equino varus of right ankle join and no other anomaly.

The placentae were examined found attached to the omentum and gut wall superficially which was separated by gentle dissection, initially massive bleeding occurred when the placenta was removed but later on the situation was under control and bleeding vessels were ligated.

The uterus, left ovary and fallopian tube ware healthy. Right ovary and tubes were attached to the placenta and distorted. A drain tube was kept in the peritoneal cavity and the abdomen was closed in layers. The patient had smooth recovery. Patient was given three units of blood transfusion. Post-operative period was uneventful and stitches were removed on the seventh post operative day (POD). Patient was discharged on 8th POD. The baby was advised to consult with an orthopedics consultants for reverse talepes equino varus of right ankle joint.

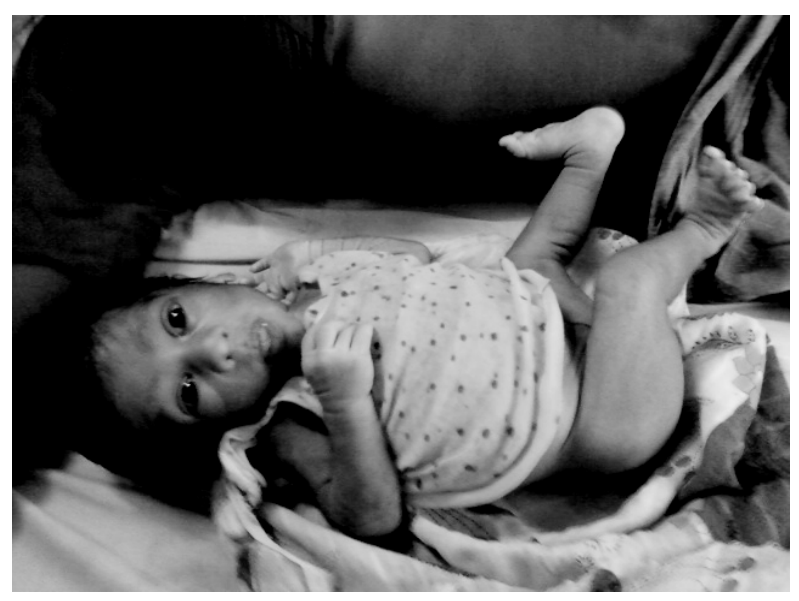

Figure -5: Baby in the third postoperative day

\section{Discussion}

AAP can be classified as being primary or secondary ${ }^{10}$. Primary AAP is the less common type, occurs when the fertilized ovum implants directly into the peritoneal cavity. Secondary AAP occurs when the fertilized ovum first implants in the fallopian tube or uterus, then due to fimbrial abortion or rupture of the fallopian tube or uterus the fetus comes to live and develop in the mother's abdominal cavity ${ }^{6,11}$.
Abdominal pregnancy is a rare obstetric complication with high maternal and even higher perinatal mortality ${ }^{12}$. Its diagnosis is difficult and a high index of suspicion is important in recognizing the condition ${ }^{2,12,13}$. None of the symptoms are individually diagnostic, so the diagnosis depends on the sum of many clues, none of which is enough by itself. A patient with an abdominal pregnancy may present with: (1) Persistent abdominal pain from about 26 to 28 weeks onwards of variable severity, which is not well localized and painful fetal movements. (2) Her "uterus' is ill-defined and the fetal parts may be abnormally easy or abnormally difficult to feel. The lie of the baby is often abnormal, and may be persistently transverse or oblique. (3) Loss of weight and general ill health. (4) Postmaturity or a dead baby which she does not expel, either spontaneously or with oxytocin. (5) Un-effaced and displaced cervix, vaginal bleeding and palpation of an abdominal mass distinct from the uterus should raise the suspicion ${ }^{14}$. Other clinical features include painful fetal movements, weight loss and vaginal bleeding. These features supported by ultrasonography made the diagnosis relatively easy. Management of abdominal pregnancy poses a clinical challenge because of risk of maternal death from hemorrhage and the possibility of congenital abnormalities ${ }^{12}$ and widely accepted treatment is immediate laparotomy because of the above complications ${ }^{13}$. However, there has been debate regarding the use of a more conservative approach if the pregnancy is discovered after 24 weeks of gestation and the fetus is alive ${ }^{15}$. This approach should only be undertaken if the patient can be kept under strict observation, preferably in hospital ${ }^{15,16}$. One of the challenging problems during laparotomy for abdominal pregnancy is risk of massive hemorrhage when attempts are made to remove the placenta ${ }^{12}$. It is advised that except the entire blood supply to the placenta can be secured with minimal risk to the patient, the placenta is best left in situ ${ }^{16}$. Use of methotrexate to hasten placental involution and resorption has been reported ${ }^{12}$.

To be considered a primary abdominal pregnancy, the pregnancy must meet the three criteria ${ }^{17,18}: 1$. Both tubes and ovaries must be in normal condition with no evidence of recent or remote injury. 2. No evidence of utero-peritoneal fistula should be found. 3. The pregnancy must be related exclusively to the peritoneal surface and be early enough to eliminate the possibility that it is a secondary implantation following a primary implantation in the tube. In our case, the three criteria for primary abdominal pregnancy were absent. So we assume that it may be a case of secondary abdominal pregnancy. 
Secondary abdominal pregnancy accounts for most cases of advanced extrauterine pregnancy. It occurs following an extrauterine tubal pregnancy that ruptures and gets re-implanted within the abdomen ${ }^{12}$. Under these circumstances, there is evidence of tubal or ovarian damage. In this report, the intermittent suprapubic pain that our patient experienced early in her pregnancy, the ultrasound examination at 30 and 38 weeks revealed 34 weeks live extra uterine pregnancy with IUGR, and the intraoperative findings of right ovary and tubes were attached to the placenta and a severely distorted right fallopian tube are highly suggestive of a tubal pregnancy that ruptured and resulted in secondary implantation on the omentum and gut wall. Accordingly, this was most likely a case of secondary abdominal pregnancy. The diagnosis was unfortunately missed during antenatal care, and the ultrasound examination findings were repeatedly misinterpreted. Owing to the site of implantation, removal of the placenta in this case was relatively easy. Since most cases of advanced abdominal pregnancy are associated with fetal demise ${ }^{2,12,19}$, delivery of live fetus at term in this case is interesting. At postnatal clinic, the patient's general condition was satisfactory. The baby had weighed $2.5 \mathrm{~kg}$ and remained grossly normal.

\section{Conclusion :}

Abdominal pregnancy is a serious and potentially life threatening condition. As abdominal pregnancy causes few symptoms, it is difficult to differentiate abdominal pregnancy from intrauterine pregnancy. Careful history taking and meticulous examination along with good ultrasonography may provide a near concluding diagnosis. Because most diagnoses of AAP are missed pre-operatively repeated abdominal pain in pregnancy needs special attention and should be one of the differential diagnosis of pregnancy with abdominal pain. The cornerstones of successful management seem to be quick intra-operative recognition, surgical skill, ready access to blood products, meticulous postoperative care and thorough assessment of the newborn.

\section{References :}

1. Brewster S. Advanced abdominal pregnancy: a case report of good maternal and perinatal outcome. West Indian med J. 2011; 60(5): 587-9.

2. Ekele BA, Ahmed Y, Nnadi D, Ishaku K. Abdominal pregnancy: ultrasound diagnosis aided by the balloon of a Foley catheter. Acta Obstet Gynecol Scand.2005;84:701-2.

3. Kassam M. Abdominal pain in pregnancy. In: David KJ, Philips JS, Carl PW, Bernard G, editors. High Risk pregnancy: Management option. London: WB Saunders; 2007.p.996-7.

4. Levent T, Eromemt M, Murat M, Murat S, Yusuf ZY. Does previous caesarean delivery increase the risk factor of ectopic pregnancy? Perinatal Journal 2005:13(2);105-9.

5. Laila A, Shuily C, Mazhar SI. Diagnostic dilemma in abdominal pregnancy A case report. Journal BCPS 2009; 177-9.

6. Amritha B, Sumangali T, Priya B, Deepak S, Sharadha R. A rare case of term viable secondary abdominal pregnancy following rupture of a rudimentary horn: a case report. J Med Case Reports 2009, 29(3):38.

7. Allibone GW, Fagan CJ, Porter SC. The sonographic features of intra abdominal pregnancy. J Clin. Ultrasound 1981; 9: 383-7.

8. Stevens CA. Malformations and deformations in abdominal pregnancy. Am J Med Genet. 1993;47:1189-95.

9. Mekki Y, Gilles JM, Mendez L, O'Sullivan MJ. Abdominal pregnancy: To remove or not to remove the placenta. Prim Care Update Ob Gyns. 1998; 5: 192.

10.Dahab AA, Aburass R, Shawkat W, Babgi R, Essa O, Mujallid RH. Full-term extrauterine abdominal pregnancy: a case report. J Med Case Reports 2011; 31(5):531.

11. Naim NM, Ahmad S, Siraj HH, Ng P, Mahdy ZA, Razi ZR. Advanced abdominal pregnancy resulting from late uterine rupture. Obstet Gynecol. 2008;111(2):502-4.

12.Gradzinskas JG. Abdominal pregnancy In: Edmond OK, editor. Dewhurst's text book of Obstetrics and Gynaecology for Postgraduates. Blackwell: Edinburgh;1999.p.70.

13.Martin JN, Sessams JK, Martin RW. Abdominal pregnancy: current concept of management. Obstet Gynecol. 1998;71:549-57.

14.King M, Bewes PC, Cairns J, Thornton J (Eds): Chapter 8. Abdominal pregnancy. In: Primary Surgery. Volume 1: nontrauma. Available from: http://www.meb.unibonn.de/dtc/primsurg/ docbook/html/x 5173 .html

15.Ifenne DI, Shittu SO, Mandara MU. Advanced abdominal pregnancy with a live fetus. Trop J Obstet Gynaecol. 1999;16:63-5.

16. Mutazedian S. Term asymptomatic abdominal pregnancy with good maternal and perinatal outcome; case report. Ir J Med Sci. 2000;25:76-80.

17. Shaw SW, Hsu JJ, Chueh HY, Hanc M, Chen FC, Chang YL, et al. Management of primary abdominal pregnancy: Twelve years of experience in a medical centre. Acta Obstetrica et Gynecologica Scandinavica 2007; 86(9):1058-62.

18. Studdiford WE. Primary peritoneal pregnancy. Am J 0bstet Gynaecol 1942; 44:487.

19. Nwobodo EI. Abdominal pregnancy: a case report. Ann Afr Med. 2004;3: 195-6. 